\title{
Tailoring a Soluble Diiron Monooxygenase for Synthesis of Aromatic $\mathrm{N}$-oxides
}

\author{
Vytautas Petkevičius ${ }^{1, *}$, Justas Vaitekūnas ${ }^{1}$, Dovydas Vaitkus ${ }^{1} \oplus$, Narimantas Čènas ${ }^{2}$ and \\ Rolandas Meškys ${ }^{1}$ (D) \\ 1 Department of Molecular Microbiology and Biotechnology, Institute of Biochemistry, Life Sciences Center, \\ Vilnius University, Saulètekio 7, LT-10257 Vilnius, Lithuania; justas.vaitekunas@bchi.vu.lt (J.V.); \\ dovydas.vaitkus07@gmail.com (D.V.); rolandas.meskys@bchi.vu.lt (R.M.) \\ 2 Department of Xenobiotics Biochemistry, Institute of Biochemistry, Life Sciences Center, Vilnius University, \\ Saulètekio 7,LT-10257 Vilnius, Lithuania; narimantas.cenas@bchi.vu.lt (N.Č.) \\ * Correspondence: vytautas.petkevicius@bchi.vu.lt
}

Received: 18 March 2019; Accepted: 3 April 2019; Published: 12 April 2019

check for updates

\begin{abstract}
The aromatic $N$-oxides have received increased attention over the last few years due to their potential application in medicine, agriculture and organic chemistry. As a green alternative in their synthesis, the biocatalytic method employing whole cells of Escherichia coli bearing phenol monooxygenase like protein PmlABCDEF (from here on - PML monooxygenase) has been introduced. In this work, site-directed mutagenesis was used to study the contributions of active site neighboring residues I106, A113, G109, F181, F200, F209 to the regiospecificity of $N$-oxidation. Based on chromogenic indole oxidation screening, a collection of PML mutants with altered catalytic properties was created. Among the tested mutants, the A113G variant acquired the most distinguishable $N$-oxidations capacity. This new variant of PML was able to produce dioxides (quinoxaline-1,4-dioxide, 2,5-dimethylpyrazine-1,4-dioxide) and specific mono- $N$-oxides (2,3,5-trimethylpyrazine-1-oxide) that were unachievable using the wild type PML. This mutant also featured reshaped regioselectivity as $N$-oxidation shifted towards quinazoline-1-oxide compared to quinazoline-3-oxide that is produced by the wild type PML.
\end{abstract}

Keywords: soluble diiron monooxygenase; aromatic $N$-oxides; protein engineering; biocatalysis; mutagenesis

\section{Introduction}

Biocatalysis has become an attractive alternative to chemical synthesis because of its exceptional selectivity, high efficiency and ability to produce relatively pure compounds. The number of biotransformation processes applied on a commercial scale is constantly increasing [1]. The usage of biocatalysts in synthesis also avoids the need for the blocking and deblocking steps often found in organic counterparts and usually are performed under mild conditions [2]. The majority of currently used enzymes in biocatalysis are hydrolases and transferases, though various oxygenases recently have been attracting tremendous interest as well [3]. These enzymes are able to hydroxylate various non-activated hydrocarbons regio- and stereospecifically, thus accomplishing chemical transformations that are of a significant challenge in synthetic chemistry [4]. Oxygenases catalyze a wide variety of reactions including activation of $\mathrm{sp}^{3}$ hybridized $\mathrm{C}$ atoms, epoxidation of $\mathrm{C}=\mathrm{C}$ double bonds, aromatic hydroxylation, $N$-oxidation, deamination and dehalogenation, Baeyer-Villiger oxidation, as well as $\mathrm{N}$-, $\mathrm{O}$ - and $\mathrm{S}$-dealkylation [5]. These enzymes can accept a diversity of substrates, including fatty acids, terpenes, steroids, prostaglandins, mono-aromatic, poly-aromatic and heteroaromatic compounds, as well as alkanes, alkenes, organic solvents, antibiotics, pesticides, carcinogens and 
toxins, thus suggesting their application in pharmacology, agriculture and bioremediation [6]. In fact, the most extensively studied cytochrome P450s together with Rieske oxygenases, flavin-dependent monooxygenases, iron- and $\alpha$-ketoglutarate-dependent dioxygenases have been applied to the synthesis of chemicals on a preparative scale [5]. Nevertheless, alternative types of oxygenases are also intensely studied. In particular, soluble diiron monooxygenases (SDIMOs) have been actively studied for a number of years. The family of SDIMO enzymes is composed of aromatic/alkene monooxygenases, phenol monooxygenases, soluble methane monooxygenases (sMMO), alkene monooxygenases, and propane monooxygenases together with tetrahydrofuran monooxygenases [7,8]. SDIMOs are threeor four-component enzyme systems which contain: i) a dimeric hydroxylase protein composed of two or three subunits in an $(\alpha \beta \gamma)_{2}$ or $(\alpha \beta)_{2}$ quaternary structure, ii) an nicotinamide adenine dinucleotide (NADH )oxidoreductase with an N-terminal chloroplast-type ferredoxin domain and a C-terminal reductase domain with flavin adenine dinucleotide (FAD) and nicotinamide adenine dinucleotide phosphate $(\mathrm{NAD}(\mathrm{P}))$-ribose binding regions, iii) a small effector or coupling protein with no prosthetic groups, and in some cases, iv) a Rieske-type ferredoxin protein. The active site of protein complex is located within the largest $\alpha$ subunit; it contains a carboxylate-bridged diiron center usually coordinated by four glutamate and two histidine residues [8]. The substrate space of those enzymes encompasses alkylated phenols, aromatic heterocycles, alkanes and alkenes of a variable length, polychlorinated hydrocarbons and other hazardous pollutants indicating their potential for bioremediation applications [9]. Taken together with favorable kinetic parameters and possible regio-, stereoselectivity, the SDIMOs emerge as candidates in various biocatalysis applications [10]. Most recently we have demonstrated that an enzyme of SDIMO family could also be employed to catalyze a different type of oxidation as phenol monooxygenase like (PML) monooxygenase transformed numerous nitrogen-containing aromatic compounds to the corresponding $N$-oxides [11]. $\mathrm{N}$-oxidation is commonly used in the chemistry of $\mathrm{N}$-heteroaromatics to increase their reactivity for further modifications [12]. In addition, $N$-oxides alone are desirable compounds in pharmacy, catalysis, agriculture and pyrotechnics [13]. Despite the array of available chemical methods for $\mathrm{N}$-oxides synthesis, biocatalytic approaches are limited and incomplete. To date, only PML catalyzed transformations have been demonstrated as a productive and green alternative to existing chemical techniques. However, an inclusive toolbox of biocatalysts for targeted and efficient preparation of desired $N$-oxides is still under development. Therefore, both directed evolution and rational design were successfully used to identify amino acids responsible for SDIMO regioselectivity and to improve their activity toward natural and artificial substrates is a promising way to improve an existing toolbox of oxygenases [14].

Thus, in this study, we analyzed sequence-structure-function based relationships in well-studied SDIMOs to create the specific PML mutants. An indirect chromogenic screening method based on indole oxidation was used to select mutants featuring new catalytic properties relative to wild type enzyme. We have successfully isolated variants of PML with altered regiospecificity for $\mathrm{N}$-oxidation, increased substrate range or capacity to produce aromatic di- $\mathrm{N}$-oxides.

\section{Results and Discussion}

\subsection{Identification of Target Amino Acids for Mutagenesis}

The PML monooxygenase shares high sequence homology to SDIMO group of phenol monooxygenases. PML shows $86 \%$ amino acids similarity to butylphenol monooxygenase from Pseudomonas putida, the closest basic local alignment search tool (BLAST) hit with known function. This PML enzyme was isolated from our collection of various oxygenases obtained from metagenomes, and its sequence is submitted to NCBI under accession number MK037457. PmlABCDEF shares all similarities to a typical SDIMO. The core of hydroxylase is composed of PmID, PmlB and PmlE, which stand for $\alpha, \beta$ and $\gamma$ subunits, respectively. PmlF is an NADH oxidoreductase, while PmlA and PmlC most likely perform regulatory functions. The carboxylate-bridged diiron center is buried within a 
catalytic PmlD subunit. The wild type PML has been introduced as a novel biocatalyst for oxidation of $\mathrm{N}$-heteroaromatic compounds [11]. It has been demonstrated that under optimal conditions $\left(30^{\circ} \mathrm{C}, \mathrm{pH}\right.$ 7.0, $24 \mathrm{~h}, 1 \mathrm{mM}$ substrate concentration) specific mono- $\mathrm{N}$-oxides are formed (Table 1 ).

The possible hotspots for mutagenesis were deduced from the MSA (multi-sequence alignment) of characterized SDIMOs (Figure 1) and the structural model of PML (Figure 2). Our first goal was to try to expand the hydrophobic pocket adjacent to the active site of PML. We expected that enlarging the active center cavity would produce mutants with preferences for bulkier and more complex substrates. Three phenylalanine residues (F181, F200, F209) situated near the diiron center in our 3D model (Figure 2) were considered as an appropriate target since previous reports indicated that amino acids in these positions have potential to change substrate specificity as well as regio- and stereospecificity [15-17]. Semiconservative phenylalanine (F181 in PML sequence; F176 in toluene 3-monooxygenase (T3MO) [18], toluene-o-xylene monooxygenase (ToMO) [15,19], toluene 4-monooxygenase (T4MO) [20]; F185 in butane monooxygenase (BMO) [21]; F172 in propene monooxygenase (PMO) [16]; F188 in soluble methane monooxygenase (sMMO) [22]; F181 in toluene 2-monooxygenase (TOM) [23,24]; F191 in HpdABCDE (Hpd) [25]) was shown to be important in determining the size of the acceptable substrate in ToMO and T4MO. Other addressed positions regulated regio- (F196 in T4MO) and stereospecificity (V188 in PMO) $[16,20]$. This hotspot varies among SDIMOs, from large aromatic residues like phenylalanine (F196 in T3MO and ToMO, F200 in TOM, F210 in Hpd) to small residues like glycine (G205 in BMO and G208 in sMMO). The corresponding position in PML is occupied by F200. Finally, mutagenesis of F205 in ToMO and T4MO demonstrated the importance of this residue, because the decreased volume of a side-chain (F205L, F205I, F205G) changed regioselectivity of the enzyme or an affinity to the specific substrate. Other SDIMOs also employ hydrophobic/aromatic residues in the analogous site (F205 in T3MO, I214 in BMO, L197 in PMO, I217 in sMMO, F209 in TOM, F219 in Hpd), including PML, which possesses F209. Keeping these data in mind, three bulky hydrophobic semiconservative amino acids of PML (F181, F200 and F209) were chosen for the site-directed mutagenesis to alanines by a classical method (Section 4, Tables 2 and 3).

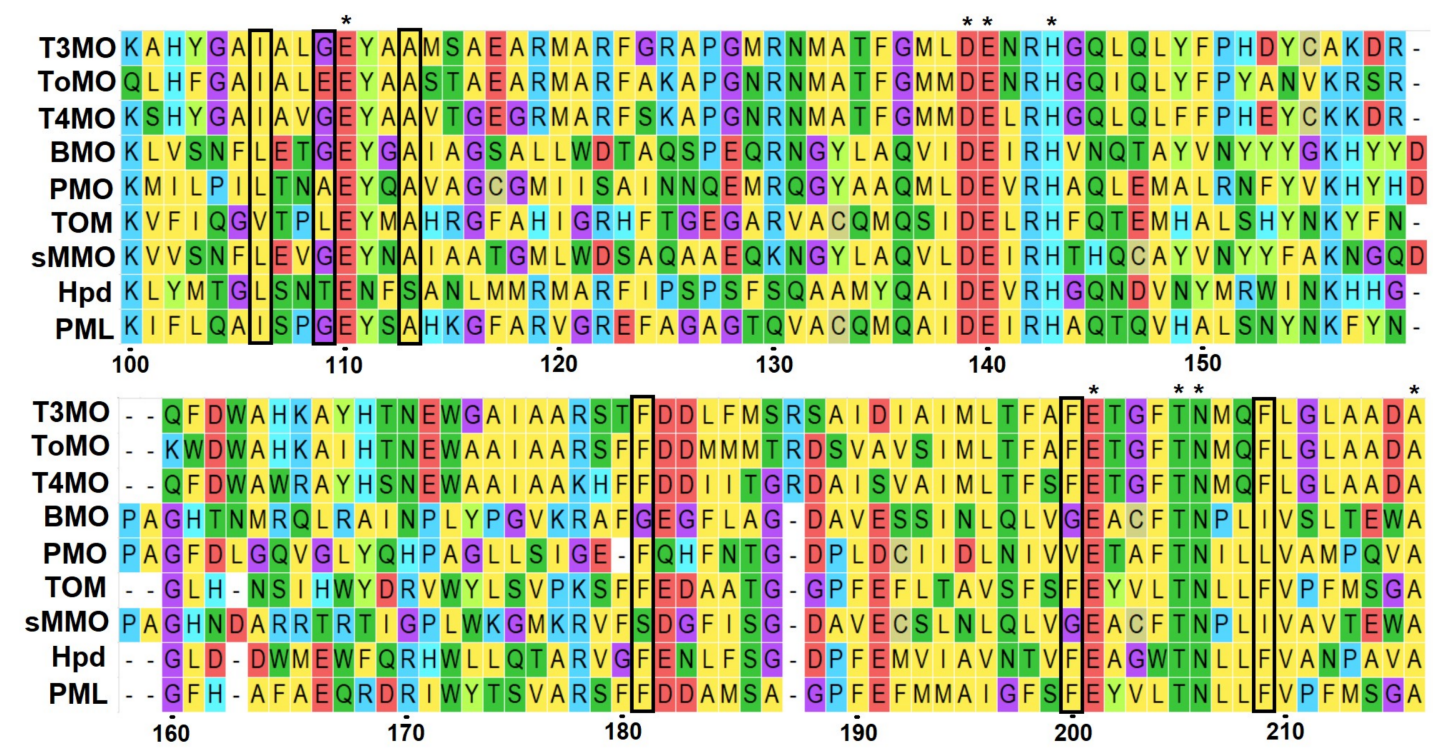

Figure 1. The protein sequence alignment between well-studied SDIMOs $\alpha$ subunits. Toluene 3-monooxygenase (T3MO) of Ralstonia picketii PKO-1, toluene-o-xylene monooxygenase (ToMO) of Pseudomonas stutzeri OX1, toluene 4-monooxygenase (T4MO) from Pseudomonas mendocina KR1, butane monooxygenase (BMO) from Thauera butanivorans, propene monooxygenase (PMO) from Mycobacterium sp. M156, toluene 2-monooxygenase (TOM) from Burkholderia cepacia G4, soluble methane monooxygenase (sMMO) from Methylococcus capsulatus Bath, 2-hydroxypyridine 5-monooxyganase HpdABCDE (Hpd) from Burkholderia sp. MAK1, PmlABCDEF (PML). The numeration is based on PML protein sequence. 
We obtained both single mutants (F181A, F200A, F209A) and double mutants (F181A/F200A, F181A/F209A, F200A/F209A) of the PML monooxygenase. Then those mutants were tested for $\mathrm{N}$-oxidation capabilities. However, only two variants (F200A and F209A) were able to transform pyridine, pyrazine, quinoxaline, quinazoline, and 2,5-dimethylpyrazine into corresponding $N$-oxides while the rest of mutants lost this activity completely. We tested F200A and F209A mutants towards a number of larger substrates (acridine, phenazine, 4,7-phenantroline, norharmane). Those compounds were poor substrates for PML and only a minor amount of $\mathrm{N}$-oxides were detected after transformation using wild type enzyme [11]. We assumed that an enlarged active site pocket of F200A and F209A mutants would result in an increased conversion compared to the wild type PML. However, the tested mutants did not possess enhanced $\mathrm{N}$-oxidation capacity of bulkier substrates. Actually, these variants seemed to retain a substrate scope of the parental enzyme with some loss in efficiency of conversion (Table 1). Similar observations were made with other monooxygenases. The equivalent mutations decreasing the volume of an active center in ToMO (F176I, F176L, F196A, F205G) showed an altered regiospecificity for phenol, toluene or naphthalene $[15,19]$. However, mutants also were less efficient as the productivity and $\mathrm{k}_{\mathrm{cat}}$ dropped for all variants. Even more, T4MO variants F176A and F196G completely lost the activity toward toluene, trichloroethane and butadiene [26]. In fact, one of the most recent studies on SDIMO engineering revealed that only the combination of saturation mutagenesis at those positions produced the best results in terms of reshaped regioseletivity and productivity [17].
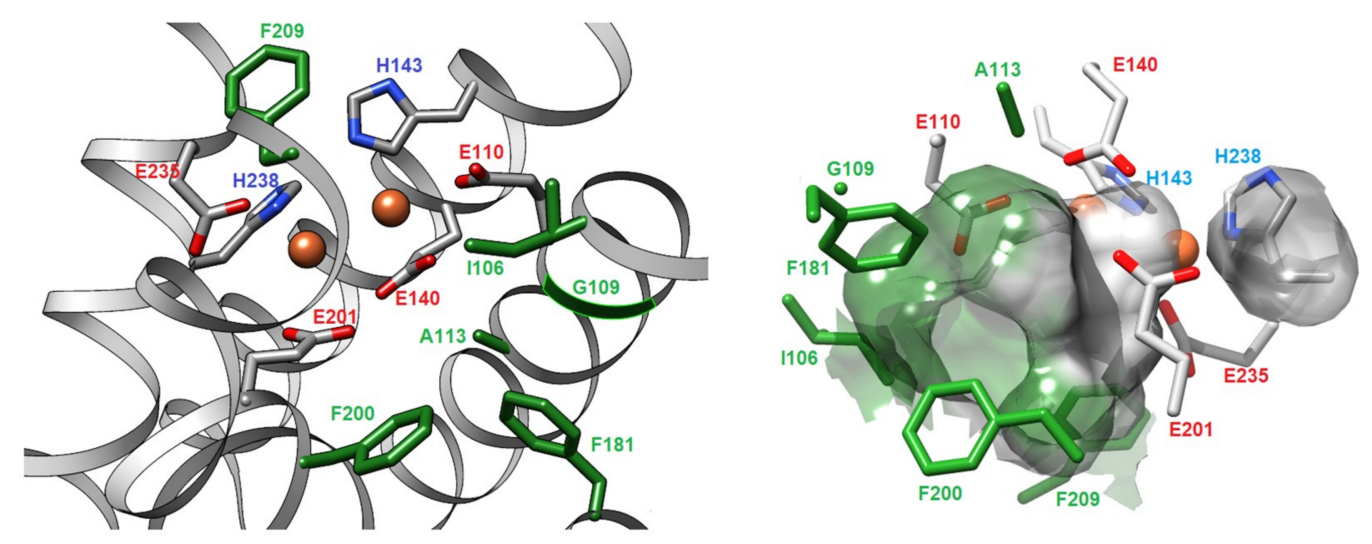

Figure 2. Two different representations of the 3D model of PmlD active site. Ribbon model (on the left) shows amino acids of interest as sticks in the helix bundle, while surface model (on the right) represents a hydrophobic pocket in the active site. The carboxylate-bridged diiron center presented in Corey-Pauling-Koltun (CPK) coloring, amino acids chosen for mutagenesis displayed in green. Orange spheres represent two iron atoms.

\subsection{The Selection of PML Mutants Based on Chromogenic Screening}

Next, we targeted other important amino acids around the active center and cross-checked with the literature anticipating more significant changes in PML substrate specificity. Three residues in PML, namely I106, A113, and G109 were selected for saturation mutagenesis for the following reasons: (i) so-called substrate gate permits the substrate to enter the active site; usually its component is an aliphatic hydrophobic amino acid (L110 in sMMO; L91 in PMO; L107 in BMO; I100 in T4MO, ToMO, T3MO; V106 in TOM; L116 in Hpd). Its substitution into a smaller residue (alanine) resulted in a relaxed regiospecificity and accessibility for larger substrates. Equally well, its replacement by a larger phenylalanine residue induced a preference for smaller substrates or even impeded the activity [22-24]. Based on MSA, the corresponding position in PML is I106; (ii) another selected hotspot in PML is A113, which is conserved in almost all analyzed sequences (A117 in sMMO; A98 in PMO; A114 in BMO; A107 in T4MO, ToMO, T3MO; A113 in TOM). This site was shown to be decisive in substrate orientation and binding $[16,18,27,28]$; (iii) an additional hotspot crucial for enzyme regioselectivity, which exhibits a great variability, e.g., E103 in ToMO [15,19], A94 in PMO [16], and G103 in T4MO [20,29] and T3MO [18]. 
Glycines are also present in BMO and sMMO (G110 and G113, respectively), although there are no data on their mutagenesis. The equivalent residue in TOM is I109, while Hpd bears T119. The corresponding amino acid in PML monooxygenase is G109.

To select potentially improved/changed enzymes from the library of mutants a convenient method was required. Aforementioned studies used a direct screening based on transformation of the appropriate substrate. The nylon membrane assay was used to visually inspect the color development by the end products (hydroquinone, resorcinol, catechol and their derivatives) produced by different mutants [19]. The PML similar approach seemed impractical as the majority of the potential $\mathrm{N}$-oxides likely are not intensely colorful and would not stain bacterial colonies. However, SDIMOs are able to convert indole into a variety of pigments (indigo, indirubin, isoindigo) and colorful hydroxyindole derivatives (isatin, 6-hydroxyindole, 7-hydroxyindole). On the other hand, an altered indole oxidation specificity after the mutagenesis of TOM, ToMO and T4MO, induced the formation of different pigments $[17,30,31]$. It enabled the selection of distinct mutants on the base of diverse color development. We hypothesized that PML mutants with an altered oxidation regiospecificity of indole might possess different $\mathrm{N}$-oxidation patterns as well. Hence, in total, nineteen differently colored colonies were selected (Figure 3). Five distinct coloration patterns were observed in the case of the I106 library. Sequencing of individual hits revealed that three different enzymes were created. I106N was selected two times, 106E was selected one time, and I106C also was selected two times. In addition, I106A mutant was created by site-direct mutagenesis separately. From the A113 library, we were able to distinguish five differently hued colonies. Sequence analysis of hits uncovered them as A113G (two times), A113V (one time) and A113F (two times). The G109 library produced the greatest coloration variety as nine different variants were selected in total. They were identified as G109M, G109Q, G109H (one time) and G109L, G109K, G109T (two times). Then those new enzymes were tested for their catalytic properties towards various $\mathrm{N}$-heteroaromatic compounds.
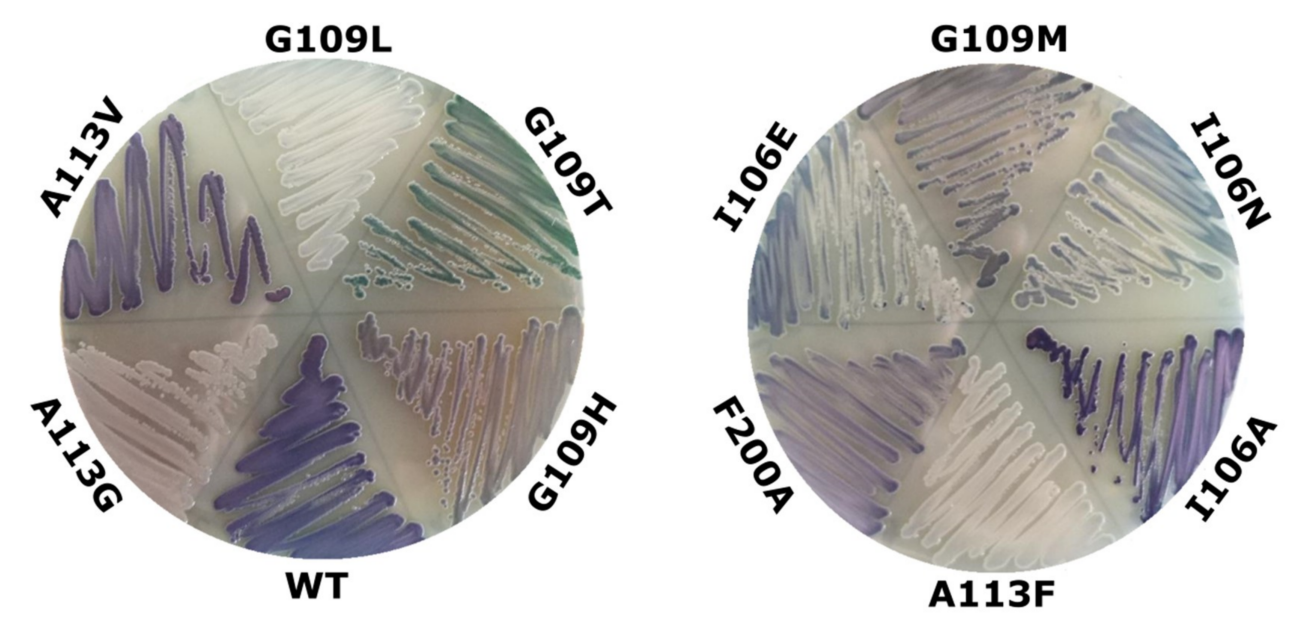

Figure 3. The formation of indigo-like pigments by isolated PML mutants. Only the most distinguishable variants are presented. Luria-Bertani (LB) plates were supplemented with $0.5 \mathrm{mM}$ indole.

\subsection{Elucidation of Properties of the Most Prominent Mutant A113G}

In order to simplify the analysis of generated mutants, we avoided the use of a wide variety of previously identified wild type PML substrates [11] and concentrated on the compounds that could yield some notable end products. Those included various diazines and triazines to investigate the possibility of multiple $\mathrm{N}$-oxidation, asymmetric compounds for potential regioselectivity shift, and some bulky derivatives that were barely oxidized by the wild type PML. From all tested mutants, the A113G variant gained the most distinctive catalytic properties (Figure 4). In particular, the A113G mutant was able to produce several di- $\mathrm{N}$-oxides which were not characteristic for the parental PML. The A113G variant transformed quinoxaline to the mixture of two products. One compound (molecular 
mass increased by $16 \mathrm{Da}$ ) was previously identified as quinoxaline-1-oxide [11]. The other product (molecular mass increased by $32 \mathrm{Da}$ ) was separated and purified in this work (Figure 4A). The Nuclear Magnetic Resonance (NMR) as well as ultraviolet (UV) spectra of the compound matched those of quinoxaline-1,4-dioxide, a prominent starting material for the synthesis of anticancer agents [13]. The similar outcome was observed for 2,5-dimethylpyrazine, which was converted to the mixture of 2,5-dimethylpyrazine-1-oxide, as in the case of the wild-type PML [11], and to a new compound 2,5-dimethylpyrazine-1,4-dioxide (Figure 4B). In contrast, parental PML produced specific dioxide solely with pyrazine (Figure $4 \mathrm{C}$ ) and only after prolonged exposure ( $48 \mathrm{~h}$ instead of $24 \mathrm{~h}$ ) and lower substrate concentration $(0.5 \mathrm{mM}$ instead of $1.0 \mathrm{mM})$.
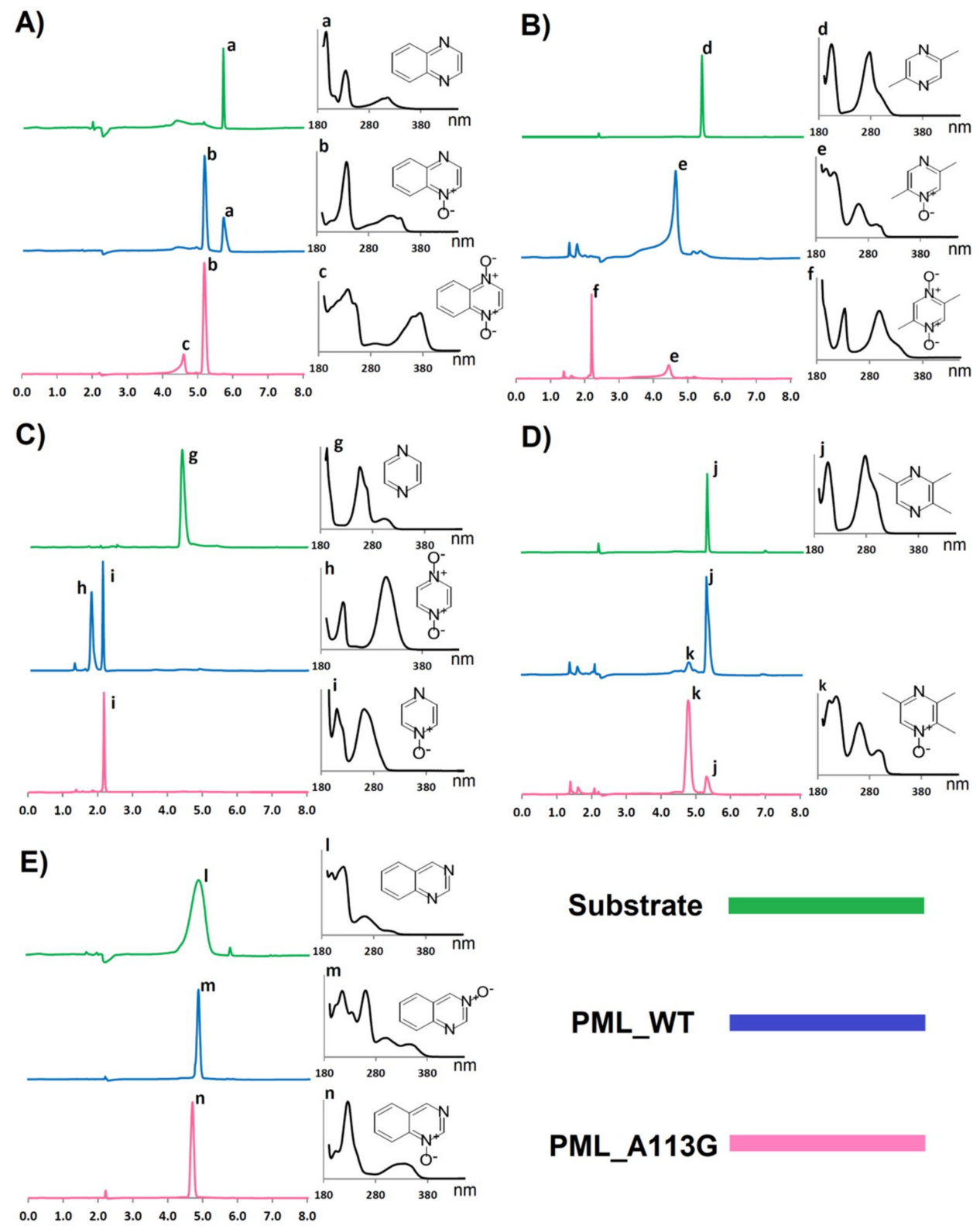

\section{Substrate \\ PML_WT \\ PML_A113G}

Figure 4. The analysis of the conversion reactions catalyzed by the wild type PML and the A113G mutant. Conversions conducted at $30^{\circ} \mathrm{C}$ for $24 \mathrm{~h}$. The initial concentration of substrate was $1.0 \mathrm{mM}$. The end products were analyzed by high-performance liquid chromatography (HPLC). A) quinoxaline (345 nm); B) 2,5-dimethylpyrazine (277 nm); C) pyrazine (282 nm); D) 2,3,5-trimethylpyrazine (260 nm); E) quinazoline $(320 \mathrm{~nm})$. Lowercase letters relate the appropriate peak in HPLC to its corresponding ultraviolet (UV) spectra. 
In addition, this mutant was able to almost completely convert 2,3,5-trimethylpyrazine (Figure 4D) to a single compound ( $92 \%$ conversion using $1.0 \mathrm{mM}$ of a substrate), which was later identified as 2,3,5-trimethylpyrazine-1-oxide. Production of latter by the wild type enzyme barely reached $6 \%$ conversion. The most distinct feature of the A113G mutant was the changed regioselectivity of $N$-oxidation. Quinazoline was oxidized by parental PML to quinazoline-3-oxide exclusively [11]. However, the A113G mutant transformed this substrate to the product with different retention time and UV spectra (Figure 4E), which indicated the formation of a new compound. After isolation and NMR analysis (Supplementary Materials), the product was confirmed as quinazoline-1-oxide, demonstrating a shift of oxygen attack from N3 to N1 position.

\subsection{Impact of the Active Site Neighboring Amino Acids in PML Mutants}

The A113 position appeared to be decisive in determining the substrate specificity. This position appears to be conservative in all well-studied monooxygenases, suggesting that it offers some evolutionary advantage. It seems that the variance in molecular volume at this site plays a key role. We showed that alanine-to-glycine substitution resulted in broadened and reshaped $N$-oxidation capabilities (Table 1). When this position was occupied by a bulkier residue (mutant A113V), the oxidation of quinoxaline and 2,3,5-trimethylpyrazine was completely suppressed. A larger substitute (A113F variant) constrained the catalysis even more since the A113F mutant just barely oxidized pyrazine, quinazoline and 2,5-dimethylpyrazine. In SDIMOs, residues acting as substrate gate were previously shown to be a major factor controlling substrate accessibility and reaction regioselectivity. However, in our study, an equivalent residue I106 of PML did not appear as important as was expected. The I106A mutant lacks leucine side-chain as a possible substrate size and entrance regulating factor. Nevertheless, there were no significant changes in oxidation patterns relative to wild type counterpart except for the lost ability to produce pyrazine-1,4-dioxide. Some other variants (I106E, I106N, I106C) also mostly retained the substrate scope of the parental enzyme, suggesting that I106 position was not critical for PML activity towards nitrogen heterocycles. Another hotspot G109 produced a variety of different PML mutants. G109M and G109Q variants apparently maintained N-oxidation features of primary PML except for the 2,3,5-trimethylpyrazine conversion. Mutants G109K, G109T and G109H additionally lost the capacity to form pyrazine-1,4-dioxide. Surprisingly, the G109L variant acquired almost exceptional ability to oxidize 2,3,5-trimethylpyrazine with a good yield, $89 \%$, despite a possibly increased molecular volume of the hydrophobic pocket. This is apparently conflicting with the properties of A113G. On the other hand, the effect of G109 modification might be indirect, as it affects the neighboring E110 which is one of the active site constituents.

Most mutations in PML seemed to act as the determinants in substrate binding or access which resulted in the decrease of the conversion efficiency for certain compounds, though led to the surge of the transformation for others. Contrary, modifications in the reviewed SDIMOs often resulted in mutants with altered regioselectivity for hydroxylation. In our case, the shift in regiospecificity was detected only for $\mathrm{N}$-oxidation of quinazoline. However, this phenomenon may be more variable using the series of compounds with a larger number of nitrogen atoms and their different surroundings. It is possible that by using substituted quinazolines, asymmetric diazines or other nitrogen-containing aromatics, these PML mutants would exhibit a broad range of different catalytic pathways. Moreover, the future modification of residues at PML active site vicinity might produce entirely diverse biocatalysts. Profoundly different from well-studied SDIMO, the monooxygenase HpdABCDE from Burkholderia sp. MAK1 serves as a good illustration. This enzyme not only transforms certain pyridines into corresponding $\mathrm{N}$-oxides, but also hydroxylates 5-position of 2-hydroxy- and 2-aminopyridine derivatives [32]. This indicates that the residues encompassing the active site of SDIMO enzymes not only determine the pathways of $N$-oxidation, but also may influence the hydroxylation of $\mathrm{N}$-heteroaromatic ring. 
Table 1. N-oxidation capabilities of PML mutants. Mono- $N$-oxide conversion * is calculated based on the consumption of the substrate. High degree of conversion $(>70 \%)$ is labeled by ' $++^{\prime}$; conversion below $10 \%$ is depicted by ' + ' sign; transformations without traceable product indicated by '-'.The ability to form a corresponding di-N-oxide is marked as 'Yes'.

\begin{tabular}{|c|c|c|c|c|c|c|c|c|c|}
\hline & $1-N \rightarrow O$ & $1,4-N \rightarrow O$ & $1-N \rightarrow O$ & $1,4-N \rightarrow \mathrm{O}$ & $1-N \rightarrow \mathrm{O}$ & $3-N \rightarrow \mathrm{O}$ & $1-N \rightarrow O$ & $1,4-N \rightarrow \mathrm{O}$ & $1-N \rightarrow 0$ \\
\hline PML_WT & ++ & Yes & ++ & - & - & ++ & ++ & - & + \\
\hline A113G & ++ & Yes & ++ & Yes & ++ & - & ++ & Yes & ++ \\
\hline A113V & ++ & Yes & - & - & - & ++ & ++ & - & - \\
\hline A113F & + & - & - & - & - & + & + & - & - \\
\hline I106A & ++ & - & ++ & - & - & ++ & ++ & - & - \\
\hline I106E & ++ & - & ++ & - & - & ++ & ++ & - & - \\
\hline $\mathrm{I} 106 \mathrm{~N}$ & ++ & Yes & + & - & - & ++ & ++ & - & - \\
\hline $\mathrm{I} 106 \mathrm{C}$ & ++ & Yes & ++ & - & - & ++ & ++ & - & - \\
\hline G109M & ++ & Yes & ++ & - & - & ++ & ++ & - & - \\
\hline G109L & ++ & - & ++ & - & - & ++ & ++ & - & ++ \\
\hline G109K & ++ & - & ++ & - & - & ++ & ++ & - & - \\
\hline G109T & ++ & - & ++ & - & - & ++ & ++ & - & - \\
\hline G109Q & ++ & Yes & ++ & - & - & ++ & ++ & - & - \\
\hline $\mathrm{G} 109 \mathrm{H}$ & ++ & - & ++ & - & - & ++ & ++ & - & - \\
\hline F181A & - & - & - & - & - & - & - & - & - \\
\hline F200A & ++ & - & + & - & - & ++ & ++ & - & - \\
\hline F209A & ++ & - & + & - & - & ++ & ++ & - & - \\
\hline F181A/F200A & - & - & - & - & - & - & - & - & - \\
\hline F181A/F209A & - & - & - & - & - & - & - & - & - \\
\hline F200A/F209A & - & - & - & - & - & - & - & - & - \\
\hline
\end{tabular}

* For conversions the whole cells of E. coli BL-21 bearing different PML mutants were used. The experiments were carried out in $10 \mathrm{~mL}$ reaction volume containing the appropriate substrate $(1 \mathrm{mM})$ at $30^{\circ} \mathrm{C}$ for $24 \mathrm{~h}$. 


\section{Conclusions}

Overall, we have demonstrated that altering the specific set of amino acids in PML affected regiospecificity of the oxygenase reaction, changed catalytic parameters or expanded range of possible substrates. We successfully applied a chromogenic screening method based on the formation of indigo-like pigments as an indirect selection platform for PML mutants with changed $\mathrm{N}$-oxidation capabilities. This study strongly contributes to the field of SDIMOs and is a promising precedent exploiting further opportunities for biooxidation of $N$-heteroaromatic compounds by these versatile catalysts.

\section{Materials and Methods}

\subsection{DNA Primers Used in this Study}

Primers used in this study were ordered from Metabion, Munich, Germany. They were designed to amplify DNA sequence in pET_pmlABCDEF plasmid flanked by restriction sites of SalI and NheI restriction endonucleases.

Table 2. DNA sequence of the primers.

\begin{tabular}{ccc}
\hline Primer Name & Primer Sequence (5' $\rightarrow \mathbf{3}^{\prime}$ ' Direction) & Use \\
\hline PheP1_Sal_F & acgcatcgtcgacgacctcttgcc & Cloning \\
PheP3_Nhe_R & Ctacagggctagcatgaactggtgg & Cloning \\
PheP3_I106A & aagatctttctgcaggccgccagcctggcgaatattc & Transforming I106 to A106 \\
PheP3_I106A_R & gaatattcgccagggctggcggcctgcagaaagatctt & Transforming I106 to A106 \\
PheP3_I106_SM & aagatctttctgcaggccNNNagccctggcgaatattc & Saturation mutagenesis of I106 \\
PheP3_I106_SM_R & gaatattcgccagggctNNNggcctgcagaaagatctt & Saturation mutagenesis of I106 \\
PheP3_G109_SM & gccatcagcctNNNgaatattccgcg & Saturation mutagenesis of G109 \\
PheP3_G109_SM_R & cgcggaatattcNNNagggctgatggc & Saturation mutagenesis of G109 \\
PheP3_A113_SM & cctggcgaatattccNNNcacaagggctttg & Saturation mutagenesis of A113 \\
PheP3_A113_SM_R & caaagcccttgtgNNNggaatattcgccagg & Saturation mutagenesis of A113 \\
PheP3_F181A_F & gtggcccgctcgttcgccgacgatgccatgag & Transforming F181 to A181 \\
PheP3_F181A_R & ctcatggcatcgtcggcgaacgagcgggccac & Transforming F181 to A181 \\
PheP3_F200A_F & ggcgatcggcttctccgccgaatatgtcctgacc & Transforming F200 to A200 \\
PheP3_F200A_R & ggtcaggacatattcggcggagaagccgatcgcc & Transforming F200 to A200 \\
PheP3_F209A_F & cctgaccaacctgctggctgtgcccttcatgtcc & Transforming F209 to A209 \\
PheP3_F209A_R & ggacatgaagggcacagccagcaggttggtcagg & Transforming F209 to A209 \\
\hline
\end{tabular}

\subsection{Plasmids and Strains Used in This Study}

In our previuos work [11], plasmid p577A was selected as a $N$-oxidation-possitive clone from metagenomic collection of various oxygenases and sequenced. PmlABCDEF gene cluster was cloned to $\mathrm{pET}$-28b expression vector (Novagen, Germany) to yield plazmid pET_pmlABCDEF.

Table 3. The characteristics of the strains and plasmids.

\begin{tabular}{|c|c|c|}
\hline Plasmid Or Strain & Relevant Characteristics & Source Or Reference \\
\hline \multicolumn{3}{|l|}{ Plasmid } \\
\hline p577A & $\begin{array}{l}\text { pUC19/HindIII + } 9 \text { kb metagenomic DNA insert containing } \\
\text { pmlABCDEF gene }\end{array}$ & [11] \\
\hline pET_pmlABCDEF & Recombinant pET-28b containing pmlABCDEF gene & [11] \\
\hline \multicolumn{3}{|l|}{ Strain } \\
\hline E. coli $\mathrm{DH} 5 \alpha$ & $\begin{array}{l}\mathrm{F}^{-} \text {endA1 glnV44 thi-1 recA1 relA1 gyrA96 deoR nup } \mathrm{purB20} \\
\varphi 80 \mathrm{~d} l a c Z \Delta \mathrm{M} 15 \Delta\left(\text { lacZYA-argF)U169, hsdR17 }\left(r_{K}{ }^{-} m_{K}{ }^{+}\right), \lambda^{-}\right.\end{array}$ & $\begin{array}{l}\text { Thermo Fischer Scientific, } \\
\text { Vilnius, Lithuania }\end{array}$ \\
\hline E. coli BL21 (DE3) & $\begin{array}{l}\mathrm{F}^{-} \text {ompT gal dcm lon } h s d S_{B}\left(r_{B}{ }^{-} m_{B}{ }^{-}\right) \lambda(\mathrm{DE} 3[\text { lacI } \\
\text { lacUV5-T7p07 ind1 sam7 nin5]) }\left[\text { malB }^{+}\right]_{\mathrm{K}-12}\left(\lambda^{S}\right)\end{array}$ & $\begin{array}{l}\text { Novagen, Darmstadt, } \\
\text { Germany }\end{array}$ \\
\hline
\end{tabular}




\subsection{Site-Directed and Saturation Mutagenesis}

Polymerase chain reaction (PCR) was conducted in $25 \mu$ of the reaction mixture containing 12.5 $\mu \mathrm{L}$ of Phusion Green Hot Start II High-Fidelity PCR Master Mix (Thermo Fischer Scientific, Vilnius, Lithuania), $200 \mathrm{nM}$ of each primer (see Section 4.1), 50 ng of matrix DNA (p577A) and water up to $25 \mu \mathrm{l}$. The mutagenesis procedure consisted of two stages. First, two overlapping PCR amplicons were produced. One was made by using PheP1_Sal_F and desired mutation reverse primer (e.g., PheP3_I106A), and the other-PheP1_Nhe_R and desired mutation forward primer (e.g., PheP3_I106A_R). For the saturation mutagenesis, a set of primers labeled 'SM' was used instead. For this PCR step, initial denaturation was $98^{\circ} \mathrm{C}$ for $30 \mathrm{~s}$ followed by 30 cycles of $98^{\circ} \mathrm{C}$ for $10 \mathrm{~s}, 55^{\circ} \mathrm{C}$ for $15 \mathrm{~s}, 72{ }^{\circ} \mathrm{C}$ for $30 \mathrm{~s}$ and a final extension at $72{ }^{\circ} \mathrm{C}$ for $7 \mathrm{~min}$. Next, these amplicons were merged by PCR to a single DNA fragment containing preferable modification. In this step initial denaturation was $98^{\circ} \mathrm{C}$ for $30 \mathrm{~s}$ followed by 30 cycles of $98^{\circ} \mathrm{C}$ for $10 \mathrm{~s}, 45-55^{\circ} \mathrm{C}$ gradient for $15 \mathrm{~s}, 72{ }^{\circ} \mathrm{C}$ for $60 \mathrm{~s}$ and a final extension at $72{ }^{\circ} \mathrm{C}$ for $7 \mathrm{~min}$. As the primers, PheP1_Sal_F and PheP3_Nhe_R were designed to amplify the region in pmlD gene flanked by SalI and NheI restriction endonucleases, thus combined PCR product was ready for cloning. The PCR amplicon was hydrolyzed with SalI and NheI (Thermo Fischer Scientific, Vilnius, Lithuania), and ligated to the properly digested plasmid pET_pmlABCDEF. Standard methods and techniques were employed for DNA manipulations (plasmid transformation, screening, isolation).

\subsection{Chromogenic Screening Assay on Agar Plates with Indole}

Mutant libraries were created by isolating total plasmid DNA from colonies of E. coli DH5 $\alpha$ transformed with the ligation mixture prepared as described in Section 4.3. Then, E. coli BL21 (DE3) cells transformed with mutant library were plated on Luria-Bertani (LB) agar containing $0.5 \mathrm{mM}$ indole and $0.5 \mathrm{mM}$ isopropyl $\beta$-D-1-thiogalactopyranoside (IPTG). Bacteria were grown overnight at $30^{\circ} \mathrm{C}$. Bacterial colonies which possessed coloration different from the wild type counterpart were picked for DNA sequencing (Macrogen, Amsterdam, Netherlands). Composition of LB media was as follows: in $\mathrm{g} / \mathrm{L}$-tryptone (Formedium, UK) 10.0, yeast extract (Merck, Germany) 5.0, NaCl (Fluka, Buchs, Switzerland) $5.0+$ agar (Formedium, Hunstanton, UK) 15.0 for LB agar plates. The medium was adjusted to $\mathrm{pH} 7.2$ and autoclaved at $1 \mathrm{~atm}$ for $30 \mathrm{~min}$.

\subsection{Conditions for biomass growth and protein biosynthesis}

E. coli BL21 (DE3) cells carrying PML monooxygenase variants were cultivated at $30^{\circ} \mathrm{C}$ on a rotary shaker at $180 \mathrm{rpm}$ in $250 \mathrm{~mL}$ flat-bottomed flasks containing $50 \mathrm{~mL}$ LB supplemented with kanamycin (40 $\mu \mathrm{g} \mathrm{mL}^{-1}$ ). The broth was additionally supplemented with glucose (to a final concentration of 1.0\%), to prevent premature recombinant protein expression. The incubation continued until $\mathrm{OD}_{600}$ reached $0.5-0.8$. Then the culture was supplemented with IPTG to a final concentration of $0.5 \mathrm{mM}$, and placed at $20^{\circ} \mathrm{C}$ on a rotary shaker at $180 \mathrm{rpm}$ overnight.

\subsection{Whole Cell Bioconversions using PML Mutants}

After induction, cells were separated from the medium by centrifugation (4000 $\times g$ for $5 \mathrm{~min})$. Then the biomass was washed twice with $10 \mathrm{mM}$ potassium phosphate buffer ( $\mathrm{pH} \mathrm{7.0)}$ and transferred (200-300 mg of wet weight) to $100 \mathrm{~mL}$ flat-bottomed flasks containing $20 \mathrm{~mL}$ of the same buffer and $1.0 \mathrm{mM}$ of the appropriate substrate. The reaction was carried out at $30^{\circ} \mathrm{C}$ on a rotary shaker at 180 $\mathrm{rpm}$. After $8 \mathrm{~h}$ of incubation, the reaction mixture was supplemented with glucose $(5 \mathrm{mmol})$, and the bioconversion was continued overnight for a total of $24 \mathrm{~h}$. Compounds used in bioconversions are listed in our previous study [11]. 


\subsection{Calculation of Bioconversion Parameters}

Conversion is expressed as $\left(\left(I_{a}-F_{a}\right) / I_{a}\right) \times 100 \%$, where $I_{a}=$ initial amount of a substrate before the conversion, and $\mathrm{F}_{\mathrm{a}}=$ final amount of a substrate after conversion. The substrate amount was determined by integrating the absorbance area ( $254 \mathrm{~nm}$ for most compounds) of a particular peak in high-performance liquid chromatography (HPLC) chromatogram. If no substrate was left, the conversion is defined as complete $(>99 \%)$.

\subsection{Isolation of the bioconversion products}

The reaction mixture was separated from biomass by centrifugation (4000 $\mathrm{g}$ for $30 \mathrm{~min}$ ). The volume of the supernatant was reduced under vacuum to a volume of $10-15 \mathrm{~mL}$, and it was transferred into a separation funnel. Then the aqueous phase was washed $>3-4$ times with the equivalent volume of organic solvent, typically chloroform. However, in order to increase extraction efficiency ethyl acetate was used for more hydrophilic $\mathrm{N}$-oxides (quinoxaline-1,4-dioxide, 2,5-dimethylpyrazine-1,4-dioxide), while toluene was applied for more hydrophobic ones (2,3,5-trimethylpyrazine-1-oxide) instead. The organic phases were combined and dried with anhydrous $\mathrm{Na}_{2} \mathrm{SO}_{4}$. The resulting solution was evaporated under reduced pressure to give a final product. All isolation stages were monitored by thin layer chromatography (TLC). The purity of the final product was verified by high-performance liquid chromatography-mass spectrometry (HPLC-MS), and the chemical structure was NMR spectroscopy.

\subsection{HPLC-MS Analysis}

The cells were separated from the reaction mixture by centrifugation. The resulting supernatant was mixed with an equal part of acetonitrile, centrifuged and analyzed. HPLC-MS analysis was performed with high-performance liquid chromatography system (Shimadzu, Japan) and a mass spectrometer (LCMS-2020, Shimadzu, Kyoto, Japan) equipped with an electrospray ionization (ESI) source. The chromatographic separation was conducted using a YMC Pack Pro C18 column, $3 \times 150 \mathrm{~mm}$ (YMC, Kyoto, Japan) at $40{ }^{\circ} \mathrm{C}$ and a mobile phase that consisted of $0.1 \%$ formic acid water solution (solvent A), and acetonitrile (solvent B) delivered in the $5 \rightarrow 95 \%$ gradient elution mode. Mass scans were measured from $\mathrm{m} / \mathrm{z} 50$ up to $\mathrm{m} / \mathrm{z} 700$, at $350^{\circ} \mathrm{C}$ interface temperature, $250^{\circ} \mathrm{CDL}$ temperature, $\pm 4,500$ $\mathrm{V}$ interface voltage, neutral DL/Qarray, using $\mathrm{N}_{2}$ as nebulizing and drying gas. Mass spectrometry data were acquired in both the positive and negative ionization modes.

\subsection{0. ${ }^{1} \mathrm{H}$ and ${ }^{13} \mathrm{C}$ NMR Data of Isolated Products}

NMR spectra were recorded in DMSO-d6 and $\mathrm{CCl}_{3} \mathrm{D}$ on an Ascend $400:{ }^{1} \mathrm{H}$ NMR $-400 \mathrm{MHz},{ }^{13} \mathrm{C}$ NMR-100 MHz (Bruker, Billerica, MA, USA). Chemical shifts ( $\delta$ ) are reported in ppm relative to the solvent resonance signal as an internal standard. The multiplicities are stated as follows: $\mathrm{s}=$ singlet, $\mathrm{d}$ $=$ doublet, $\mathrm{dd}=$ doublet of doublets, $\mathrm{t}=$ triplet, $\mathrm{m}=$ multiplet.

2,6-Dimethylpyrazine-1,4-dioxide was isolated as a white solid. ${ }^{1} \mathrm{H}$ NMR (400 MHz, DMSO-d6): $\delta=2.26$ (s, 6H, CH3), 8.50 (s, 2H, CH). ${ }^{13} \mathrm{C}$ NMR (100 MHz, DMSO-d6): $\delta=14.3,135.3,144.4$.

2,3,5,-Trimethylpyrazine-1-oxide was isolated as a brown liquid. ${ }^{1} \mathrm{H}$ NMR (400 MHz, DMSO-d6): $\delta=2,30-2,48(\mathrm{~m}, 9 \mathrm{H}, \mathrm{CH} 3), 8.31(\mathrm{~s}, 1 \mathrm{H}, \mathrm{CH}) .{ }^{13} \mathrm{C}$ NMR (100 MHz, DMSO-d6): $\delta=21.0,22.4,22.5,143.3$, 152.6, 153.3, 155.1 .

Quinoxaline-1,4-dioxide was isolated as an orange solid. ${ }^{1} \mathrm{H}$ NMR (400 MHz, DMSO-d6): $\delta=7.95-8.05$ $(\mathrm{m}, 2 \mathrm{H}, \mathrm{CH}), 8.43-8.52(\mathrm{~m}, 2 \mathrm{H}, \mathrm{CH}), 8.54(\mathrm{~s}, 2 \mathrm{H}, \mathrm{CH}) .{ }^{13} \mathrm{C} \mathrm{NMR}\left(100 \mathrm{MHz}, \mathrm{CCl}_{3} \mathrm{D}\right): \delta=120.6,130.4$, 132.2, 138.6.

Quinazoline-1-oxide was isolated as white solid. ${ }^{1} \mathrm{H}$ NMR (400 MHz, DMSO-d6): $\delta=7.89-7.95$ (dd, $J=7.9,7.2 \mathrm{~Hz}, 1 \mathrm{H}, \mathrm{CH}), 8.07-8.14(\mathrm{dd}, J=8.1,7.8 \mathrm{~Hz}, 1 \mathrm{H}, \mathrm{CH}), 8.30(\mathrm{~d}, J=8.2 \mathrm{~Hz}, 1 \mathrm{H}, \mathrm{CH}), 8.47(\mathrm{~d}$, $J=8.7,1 \mathrm{H}, \mathrm{CH}), 9.14(\mathrm{~s}, 1 \mathrm{H}, \mathrm{CH}), 9.31(\mathrm{~s}, 1 \mathrm{H}, \mathrm{CH}) .{ }^{13} \mathrm{C}$ NMR $(100 \mathrm{MHz}, \mathrm{DMSO}-\mathrm{d} 6): \delta=118.3,128.6$, $130.7,135.4,143.4,147.4$. 


\subsection{PML Protein 3D Modelling}

The 3D model of PML was generated with I-TASSER server [33], Chimera 1.13.1 was used for presentation [34].

Supplementary Materials: The following are available online at http://www.mdpi.com/2073-4344/9/4/356/s1: ${ }^{1} \mathrm{H}$ NMR and ${ }^{13} \mathrm{C}$ NMR spectra of synthesized compounds.

Author Contributions: Investigation, data analysis, draft preparation, V.P.; data analysis, writing-review and editing, J.V.; investigation, writing — review and editing, D.V.; writing—review and editing, supervision, R.M.; writing-review and editing, supervision, N.Č.

Funding: This work was supported by the European Social Fund according to the activity 'Improvement of researchers' qualification by implementing world-class R\&D projects' of Measure No. 09.3.3-LMT-K-712 (grant No. DOTSUT-34(09.3.3-LMT-K712-01-0058/LSS-600000-58)). This research was performed in cooperation with the INMARE consortium.

Acknowledgments: V.P., J.V. and N.Č. gratefully acknowledge the support of the European Social Fund.

Conflicts of Interest: The authors declare no conflict of interest.

\section{References}

1. Badenhorst, C.P.S.; Bornscheuer, U.T. Getting momentum: From biocatalysis to advanced synthetic biology. Trends Biochem. Sci. 2018, 43, 180-198. [CrossRef] [PubMed]

2. Hughes, G.; Lewis, J.C. Introduction: Biocatalysis in Industry. Chem. Rev. 2018, 118, 1-3. [CrossRef]

3. Turner, N.J.; Kumar, R. Editorial overview: Biocatalysis and biotransformation: The golden age of biocatalysis. Curr. Opin. Chem. Biol. 2018, 43, A1-A3. [CrossRef]

4. Holtmann, D.; Fraaije, M.W.; Arends, I.W.; Opperman, D.J.; Hollmann, F. The taming of oxygen: Biocatalytic oxyfunctionalisations. Chem. Commun. 2014, 87, 13180-13200. [CrossRef]

5. King-Smith, E.; Zwick, C.R., 3rd; Renata, H. Applications of oxygenases in the chemoenzymatic total synthesis of complex natural products. Biochemistry 2018, 57, 403-412. [CrossRef]

6. Prier, C.K.; Kosjek, B. Recent preparative applications of redox enzymes. Curr. Opin. Chem. Biol. 2018, 49, 105-112. [CrossRef] [PubMed]

7. Coleman, N.V.; Bui, N.B.; Holmes, A.J. Soluble di-iron monooxygenase gene diversity in soils, sediments and thane enrichments. Environ. Microbiol. 2006, 8, 1228-1239. [CrossRef]

8. Leahy, J.G.; Batchelor, P.J.; Morcomb, S.M. Evolution of the soluble diiron monooxygenases. FEMS Microbiol. Rev. 2003, 27, 449-479. [CrossRef]

9. Holmes, A.J. The diversity of doluble di-iron monooxygenases with bioremediation applications. In Advances in Applied Bioremediation; Singh, A., Kuhad, R., Ward, O., Eds.; Springer: Berlin, Germany, 2009; Volume 17.

10. Notomista, E.; Scognamiglio, R.; Troncone, L.; Donadio, G.; Pezzella, A.; Di Donato, A.; Izzo, V. Tuning the specificity of the recombinant multicomponent toluene $o$-xylene monooxygenase from Pseudomonas sp. Strain OX1 for the biosynthesis of tyrosol from 2-phenylethanol. Appl. Environ. Microbiol. 2011, 77, 5428-5437. [CrossRef] [PubMed]

11. Petkevičius, V.; Vaitekūnas, J.; Tauraitė, D.; Stankevičiūtė, J.; Šarlauskas, J.; Čènas, N.; Meškys, R. A biocatalytic synthesis of heteroaromatic $N$-oxides by whole cells of Escherichia coli expressing the multicomponent, soluble di-iron monooxygenase (SDIMO) PmlABCDEF. Adv. Synth. Catal. 2019, 361. [CrossRef]

12. Clayden, J.; Greeves, N.; Warren, S.G. Aromatic heterocycles 1: Reactions. In Organic Chemistry, 2nd ed.; Oxford University Press: New York, NY, USA, 2012; pp. 730-731.

13. Mfuh, A.M.; Larionov, O.V. Heterocyclic $N$-oxides-An emerging class of therapeutic agents. Curr. Med. Chem. 2015, 22, 819-857. [CrossRef]

14. Nichol, T.; Murrell, J.C.; Smith, T.J. Controlling the activities of the diiron centre in bacterial monooxygenases: Lessons from mutagenesis and biodiversity. Eur. J. Inorg. Chem. 2015, 2015, 3419-3431. [CrossRef]

15. Notomista, E.; Cafaro, V.; Bozza, G.; Di Donato, A. Molecular determinants of the regioselectivity of toluene/o-xylene monooxygenase from Pseudomonas sp. Strain OX1. Appl. Environ. Microbiol. 2009, 75, 823-836. [CrossRef] 
16. Chan Kwo Chion, C.K.; Askew, S.E.; Leak, D.J. Cloning, expression, and site-directed mutagenesis of the propene monooxygenase genes from Mycobacterium sp. Strain M156. Appl. Environ. Microbiol. 2005, 71, 1909-1914. [CrossRef]

17. Sönmez, B.; Yanık-Yıldırım, K.C.; Wood, T.K.; Vardar-Schara, G. The role of substrate binding pocket residues phenylalanine 176 and phenylalanine 196 on Pseudomonas sp. OX1 toluene $o$-xylene monooxygenase activity and regiospecificity. Biotechnol. Bioeng. 2014, 111, 1506-1512. [CrossRef]

18. Fishman, A.; Tao, Y.; Rui, L.; Wood, T.K. Controlling the regiospecific oxidation of aromatics via active site engineering of toluene para-monooxygenase of Ralstonia pickettii PKO1. J. Biol. Chem. 2005, 280, 506-514. [CrossRef]

19. Vardar, G.; Wood, T.K. Protein engineering of toluene-o-xylene monooxygenase from Pseudomonas stutzeri OX1 for synthesizing 4-methylresorcinol, methylhydroquinone, and pyrogallol. Appl. Environ. Microbiol. 2004, 70, 3253-3262. [CrossRef]

20. Pikus, J.D.; Studts, J.M.; McClay, K.; Steffan, R.J.; Fox, B.G. Changes in the regiospecificity of aromatic hydroxylation produced by active site engineering in the diiron enzyme toluene 4-monooxygenase. Biochemistry 1997, 36, 9283-9289. [CrossRef]

21. Halsey, K.H.; Sayavedra-Soto, L.A.; Bottomley, P.J.; Arp, D.J. Site-directed amino acid substitutions in the hydroxylase alpha subunit of butane monooxygenase from Pseudomonas butanovora: Implications for substrates knocking at the gate. J. Bacteriol. 2006, 188, 4962-4969. [CrossRef]

22. Borodina, E.; Nichol, T.; Dumont, M.G.; Smith, T.J.; Murrell, J.C. Mutagenesis of the "leucine gate" to explore the basis of catalytic versatility in soluble methane monooxygenase. Appl. Environ. Microbiol. 2007, 73, 6460-6467. [CrossRef]

23. Rui, L.; Kwon, Y.M.; Fishman, A.; Reardon, K.F.; Wood, T.K. Saturation mutagenesis of toluene ortho-monooxygenase of Burkholderia cepacia G4 for Enhanced 1-naphthol synthesis and chloroform degradation. Appl. Environ. Microbiol. 2004, 70, 3246-3252. [CrossRef]

24. Canada, K.A.; Iwashita, S.; Shim, H.; Wood, T.K. Directed evolution of toluene ortho-monooxygenase for enhanced 1-naphthol synthesis and chlorinated ethane degradation. J. Bacteriol. 2002, 184, 344-349. [CrossRef]

25. Petkevičius, V.; Vaitekūnas, J.; Stankevičiūtè, J.; Gasparavičiūtè, R.; Meškys, R. Catabolism of 2-hydroxypyridine by Burkholderia sp. Strain MAK1: A 2-hydroxypyridine 5-monooxygenase encoded by hpdABCDE catalyzes the first step of biodegradation. Appl. Environ. Microbiol. 2018, 84, e00387-18. [CrossRef]

26. Steffan, R.J.; McClay, K.R. Preparation of Enantio-Specific Epoxides; Shaw Environmental \& Infrastructure, Inc.: Washington, DC, USA, 2000.

27. Tao, Y.; Fishman, A.; Bentley, W.E.; Wood, T.K. Altering toluene 4-monooxygenase by active-site engineering for the synthesis of 3-methoxycatechol, methoxyhydroquinone, and methylhydroquinone. J. Bacteriol. 2004, 186, 4705-4713. [CrossRef]

28. Carlin, D.A.; Bertolani, S.J.; Siegel, J.B. Biocatalytic conversion of ethylene to ethylene oxide using an engineered toluene monooxygenase. Chem. Commun. 2015, 51, 2283-2285. [CrossRef]

29. Mitchell, K.H.; Studts, J.M.; Fox, B.G. Combined participation of hydroxylase active site residues and effector protein binding in a para to ortho modulation of toluene 4-monooxygenase regiospecificity. Biochemistry 2002, 41, 3176-3388. [CrossRef]

30. McClay, K.; Boss, C.; Keresztes, I.; Steffan, R.J. Mutations of toluene-4-monooxygenase that alter regiospecificity of indole oxidation and lead to production of novel indigoid pigments. Appl. Environ. Microbiol. 2005, 71, 5476-5483. [CrossRef]

31. Rui, L.; Reardon, K.F.; Wood, T.K. Protein engineering of toluene ortho-monooxygenase of Burkholderia cepacia G4 for regiospecific hydroxylation of indole to form various indigoid compounds. Appl. Microbiol. Biotechnol. 2005, 66, 422-429. [CrossRef]

32. Stankevičiūtè, J.; Vaitekūnas, J.; Petkevičius, V.; Gasparavičiūtè, R.; Tauraitè, D.; Meškys, R. Oxyfunctionalization of pyridine derivatives using whole cells of Burkholderia sp. MAK1. Sci. Rep. 2016, 6, 39129. [CrossRef] 
33. Zhang, Y. I-TASSER server for protein 3D structure prediction. BMC Bioinform. 2008, 9, 40. [CrossRef]

34. Pettersen, E.F.; Goddard, T.D.; Huang, C.C.; Couch, G.S.; Greenblatt, D.M.; Meng, E.C.; Ferrin, T.E. UCSF Chimera-A visualization system for exploratory research and analysis. J. Comput. Chem. 2004, 25, 1605-1612. [CrossRef] 